\title{
Real-Time Capturing of the Nuclear Wave-Packet Shape in Self-Trapped Excitons
}

\author{
Taira Matsuoka, ${ }^{1}$ Jun Takeda, ${ }^{2}$ Susumu Kurita, ${ }^{2}$ and Tohru Suemoto ${ }^{1}$ \\ ${ }^{1}$ Institute for Solid State Physics, The University of Tokyo, Kashiwanoha 5-1-5, Kashiwa-shi, Chiba, 277-8581, Japan \\ ${ }^{2}$ Faculty of Engineering, Yokohama National University, Yokohama, 240-8501, Japan
}

(Received 9 July 2003; published 9 December 2003)

\begin{abstract}
We measured the time evolutions of the luminescence spectra in a quasi-1D halogen-bridged platinum complex $\left[\mathrm{Pt}(\mathrm{en})_{2}\right]\left[\mathrm{Pt}(\mathrm{en})_{2} \mathrm{Br}_{2}\right]\left(\mathrm{ClO}_{4}\right)_{4}$ with a 50 fs resolution up-conversion spectroscopy and succeeded in real-time visualization of the shape of the wave packet (WP) propagating on the adiabatic potential surface of a self-trapped exciton. The behavior of the WP is in excellent agreement with a calculation based on a harmonic oscillator. We have found that the damping of the WP oscillation is highly nonexponential.
\end{abstract}

DOI: 10.1103/PhysRevLett.91.247402

PACS numbers: 78.47.+p, 71.35.Aa, 78.55.Hx

In understanding the ultrafast dynamics of the atomic motions in molecules and solids from a viewpoint of quantum mechanics, it is essentially important to know the behavior of the wave packet (WP), which is expressed as a coherent superposition of vibrational eigenfunctions of the system. Real-time direct access to the shape of WP in the intermediate states is inevitable for clarifying the details of the dynamics and designing the coherent control of the chemical processes. In polyatomic molecules, the change of the atomic configurations will be described as a propagation of the WP on a multidimensional adiabatic surface. During propagation, the WP will suffer decoherence and energy dissipation, and in consequence its shape will be changed as time elapses. The WP oscillation on the adiabatic potential curve was experimentally observed by Zewail's group for the first time in $\mathrm{NaI}$ molecules in a gas phase $[1,2]$. In solids, the situation is more complicated, because they have an infinite degree of freedom. The oscillations in solids were observed in $F$ centers [3] and in self-trapped excitons (STE) of $\mathrm{NaCl}$ [4]. However, it was hard to elucidate the shape of the WP, because it was monitored at one or a few points on the configuration coordinate. In this Letter, we report the first complete capturing of the time-evolving shape of the WP oscillating in an adiabatic potential well. The behavior of the WP shape is excellently described in terms of a harmonic oscillator. However, the highly nonlinear damping behavior is considered to be phenomena characteristic to solids.

We investigated a single crystal of a quasi-onedimensional halogen-bridged platinum complex $\left[\mathrm{Pt}(\mathrm{en})_{2}\right]\left[\mathrm{Pt}(\mathrm{en})_{2} \mathrm{Br}_{2}\right]\left(\mathrm{ClO}_{4}\right)_{4}$ ("en" means ethylenediamine). The crystal is composed of one-dimensional (1D) chains of alternating platinum and halogen ions [5]. The electronic structure in the ground state is expressed as the repetition of the component $\cdots-\mathrm{Pt}^{3+\rho}-$ $\mathrm{Br}^{-}-\mathrm{Pt}^{3-\rho}-\mathrm{Br}^{-}-\cdots(0<\rho<1)$, which is a commensurate charge density wave originating from Peierls instability. Light excitation corresponding to a charge transfer $(\mathrm{CT})$ transition from $\mathrm{Pt}^{3-\rho}$ sites to $\mathrm{Pt}^{3+\rho}$ sites leads to a generation of free excitons (FEs). The FEs immediately relax to STEs accompanying lattice relaxation, because there no energy barrier exists between FE and STE states in 1D systems. The configuration of the STE is $\mathrm{Pt}^{3+}-\mathrm{Br}^{-}-\mathrm{Pt}^{3+}$ as shown in the inset of Fig. 1. The wave function and the lattice deformation are believed to be localized on several atoms. In the previous reports by Tomimoto et al. [6,7] the oscillation of the WP was observed but the shape of the WP was not discussed,

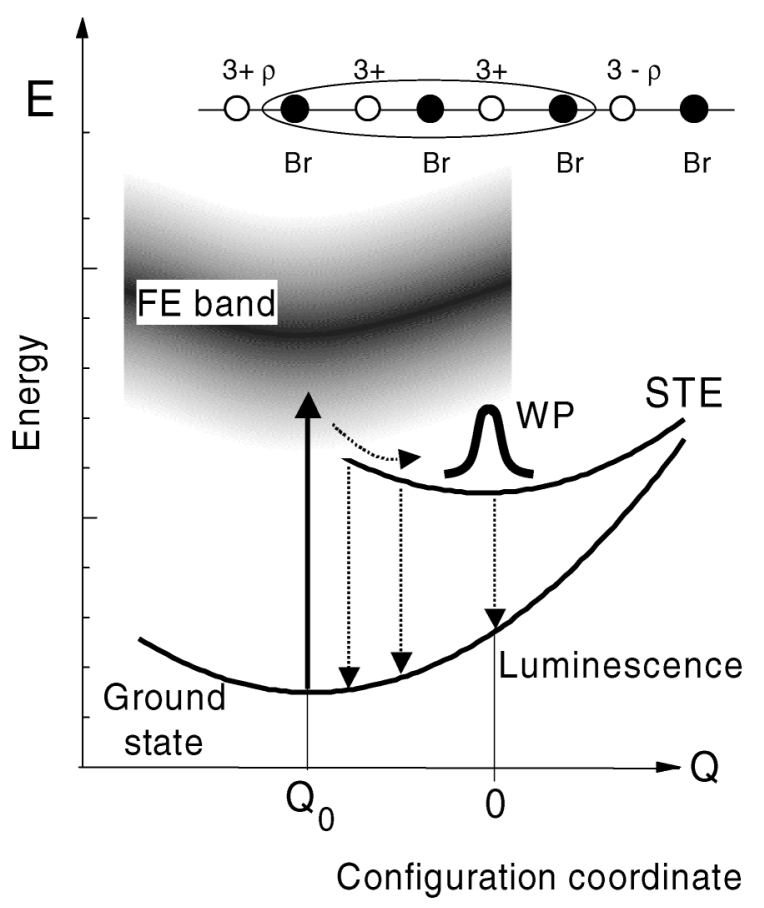

FIG. 1. Adiabatic potential curves for ground state and the lowest state of the self-trapped exciton. The abscissa represents the configuration coordinate, which approximately corresponds to the displacement of the $\mathrm{Br}$ ion. The photon energies of the luminescence are represented by the lengths of downward arrows. The inset is a schematic display of the linear chain of the halogen-bridged platinum complex. The chain shows the self-trapped exciton consisting of two $\mathrm{Pt}^{3+}$ ions. 
because of experimental difficulties in deciding the precise time origin and the relative intensities of the luminescence at different photon energies.

We measured the time evolution of luminescence at room temperature by means of the frequency up-conversion technique, using a mode-locked Ti-sapphire laser with a repetition rate of $76 \mathrm{MHz}$, a photon energy $1.6 \mathrm{eV}$, and a pulse width $\sim 25 \mathrm{fs}$. The light was separated into two beams. One beam was the gate beam, and the other was used as the pump beam to excite the sample. The pump beam was polarized parallel to the $1 \mathrm{D}$ chains of the sample. The luminescence was focused onto a nonlinear optical crystal $\left(0.3 \mathrm{~mm}\right.$-thick $\left.\mathrm{LiIO}_{3}\right)$ by a pair of off-axis paraboloidal mirrors and mixed with the variably delayed gate pulses. The signal of the frequency up-converted light was analyzed by a double-grating monochromator and detected by a standard photon counting system. The highest overall time resolution of this system was $40 \mathrm{fs}$ [8], which we believe is the best value ever achieved. Time origin was defined as the center point of the crosscorrelation trace. The spectral response of the total upconversion system (up-conversion efficiency) has been calibrated by taking the up-conversion signal of a tungsten standard lamp. The accuracy of the time origin and the reproducibility of the up-conversion efficiency were assured by controlling the $\mathrm{LiIO}_{3}$ crystal by a computer with accuracies of $0.5 \mu \mathrm{m}$ in position and $0.005^{\circ}$ in angle.

Figure 2(a) shows the time evolutions of the luminescence intensity under $1.6 \mathrm{eV}$ light excitation with an average power of $1.0 \mathrm{~mW}$. This energy corresponds to the low energy side of the CT band. The dynamics was investigated with a time resolution of $50 \mathrm{fs}$ at time inter-
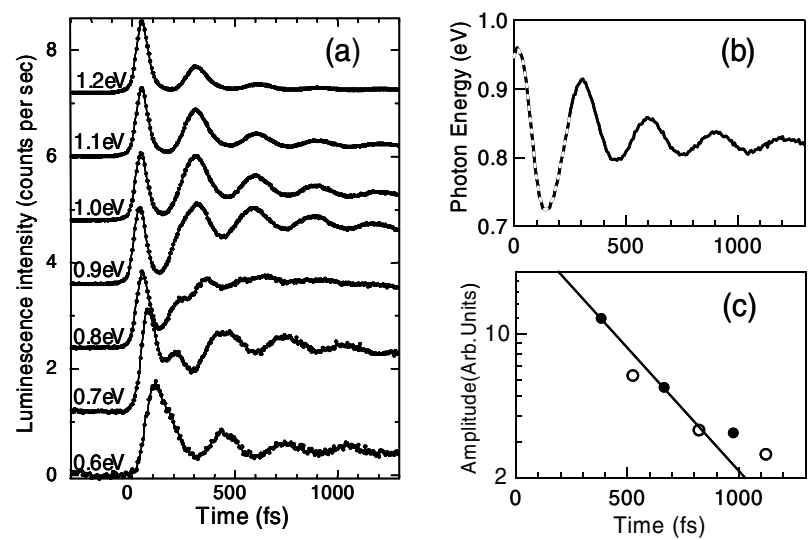

FIG. 2. (a) Time evolutions of the luminescence intensity under $1.6 \mathrm{eV}$ excitation. The average power of the gate beam was $100 \mathrm{~mW}$ and of the pump beam $1 \mathrm{~mW}$. The ordinate shows the calibrated luminescence intensity. (b) Time evolution of the first moment of the luminescence spectra. The curve is reliable only after $250 \mathrm{fs}$ (see text). (c) Peak-to-dip (solid circles) and dip-to-peak (open circles) amplitude of the first moment for each half cycle plotted on a semilogarithmic scale. The line corresponds to the decay constant of $365 \mathrm{fs}$. vals of 6.7 fs. The ordinate of Fig. 2(a) shows the calibrated luminescence intensity (photons per second per unit energy interval). The luminescence intensity oscillates at a period of about $300 \mathrm{fs}$ at $1.2 \mathrm{eV}$, reflecting the $\mathrm{WP}$ motion on the adiabatic potential curve shown in Fig. 1. At the photon energy of $0.8 \mathrm{eV}$ (peak of luminescence spectrum [9]) in Fig. 2(a), we can see the overtone oscillation with a period of about $150 \mathrm{fs}$, which is onehalf of the period of the WP oscillation. This is because the WP passes through the potential minimum every $150 \mathrm{fs}$ in alternate directions. Moreover, looking at Fig. 2(a), we can observe the antiphasing behavior between the oscillations at 0.7 and $0.9 \mathrm{eV}$. The luminescence below and above $0.8 \mathrm{eV}$ probes the $\mathrm{WP}$ at the points on the opposite sides of the potential minimum on the adiabatic potential curve. This clearly shows that the WP is moving back and forth around the potential minimum.

We then followed with the visualization of the explicit shape of the oscillating WP. As shown in Fig. 3, we plot the $7 \times 240$ data in Fig. 2(a) as photon energy versus time delay. Luminescence intensity is depicted with a gray scale. The data between the experimental points were estimated by linear interpolation. We can clearly see the sinusoidal motion of the WP around $0.8 \mathrm{eV}$ from 80 fs to 1300 fs. The amplitude of the first downward swing is slightly larger than $0.2 \mathrm{eV}$. If we assume a parabolic potential shape with the same curvature for the ground state and the excited state, the luminescence photon energy $E$ is linearly related to the configuration coordinate $Q$ as

$$
Q=\alpha\left(E_{0}-E\right)
$$

where $E_{0}$ is the photon energy at the luminescence peak, and $\alpha$ is a positive constant. The origin of $Q$ is taken at the potential minimum of the excited state (Fig. 1). In the case of this sample, we can regard the configuration coordinate as the displacement of halogen ions, because the platinum ions are rather rigidly fixed to the framework, which consists of the perchlorate ions and ethylenediamine ligands [5]. In short, we can consider Fig. 3 as

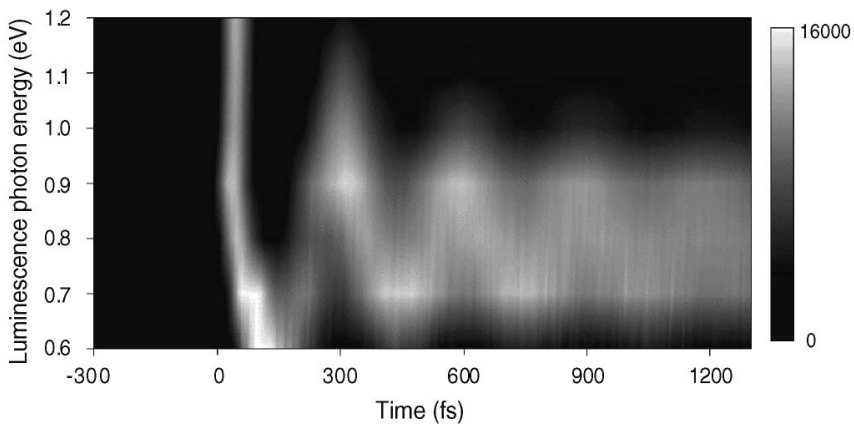

FIG. 3. Two dimensional display of the time evolution of the luminescence intensity. The ordinate is the luminescence photon energy, which approximately corresponds to the spatial position of the $\mathrm{Br}$ ion. The brighter part has larger amplitude. 
the trajectory of $\mathrm{Br}^{-}$ions. The ions keep equilibrium positions in the ground state. The CT excitation by photons generates FEs with the electron and hole wave functions spread over the crystal. In the strong electron-lattice coupling system, the propagating excitons end up with localizing somewhere on the 1D chain and the ions begin damping oscillations around the new equilibrium positions because the equilibrium positions of ions are changed.

The vertical cross sections of Fig. 3 give us the luminescence spectra, i.e., the shape of the WP, at an arbitrary time. Though we obtained 240 spectra, here we show only several characteristic ones in Fig. 4(a). The upper six frames were taken every $40 \mathrm{fs}$. Because the squared WP amplitude is proportional to the luminescence intensity, transient luminescence spectra directly reflect the shape of the WP at each time. As a result, we can see not only the swing of the WP but also the change of its shape. At $87 \mathrm{fs}$ in the first downward swing, the left edge of the WP is quite sharp. At 127 and $167 \mathrm{fs}$, the peak of the WP appears located beyond the lower limit of the measured energy range. The peak comes back in sight again at $207 \mathrm{fs}$. During the movement to the left across the center of oscillation, the peak intensity decreases gradually. However, it increases again at the left turning point around $287 \mathrm{fs}$ and the shape becomes asymmetric. The lower four frames in Fig. 4(a) show the oscillation after this fast damping. The small amplitude oscillation lasts till the end of the scan at 1300 fs. One of the most important features is that the peak intensity is higher around the turning points $(127,287-307$, and $453 \mathrm{fs}$ ) than around the center of oscillation.

The time evolution of the WP amplitude observed at fixed $Q$ has been already analyzed in terms of a model based on the interaction mode of phonons [7]. In this model, the interaction mode consists of the linear superposition of bulk phonon modes and the lattice relaxation towards the STE occurs along this mode [10]. This analysis seemed plausible, because the time evolution of the luminescence intensity measured with a $90 \mathrm{fs}$ resolution was reasonably well reproduced [7]. However the model assumed the Gaussian shape for the WP all the way during relaxation, which is evidently inadequate in understanding the time dependence of the WP shape. Therefore, we use the model which expresses a correct WP shape but does not include the damping effect. In the following, we calculate the time-dependent WP shape $\Psi(t, Q)$ by superposing the well-known wave functions of the harmonic oscillator,

$$
\begin{gathered}
\Psi(t, Q)=\sum_{n} c_{n} \psi_{n}(Q) \exp [i \hbar \omega n t], \\
\psi_{n}(Q)=\sqrt{\frac{1}{\left(2^{n} n ! \sqrt{\pi}\right) b}} H_{n}(Q / b) \exp \left(-\frac{1}{2}(Q / b)^{2}\right),
\end{gathered}
$$

where $n$ is the index of vibrational levels, $b$ is a con- (a)

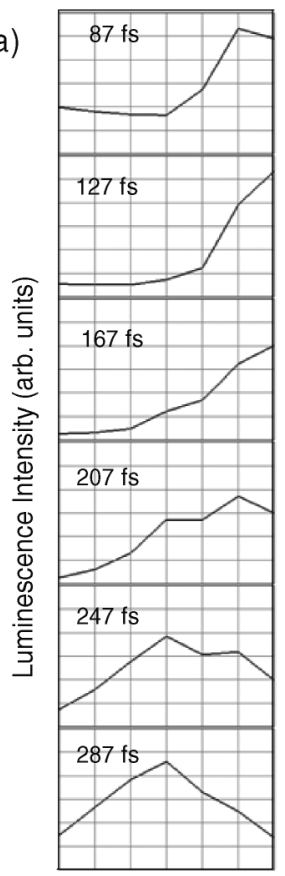

(b)

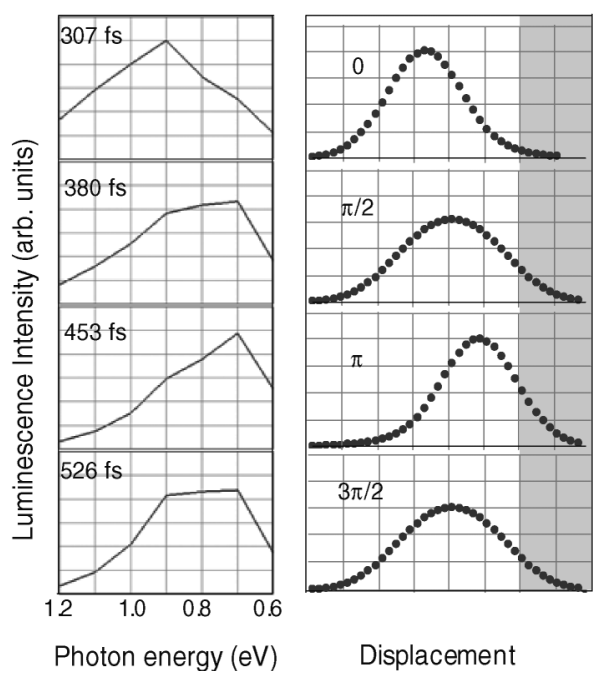

FIG. 4. Time evolutions of the wave packet. The curves in column (a) are the vertical cross sections cut from the 2D plot shown in Fig. 3. The upper six frames are taken every 40 fs just after excitation. The lower four frames are taken every $73 \mathrm{fs}$. The plots in column (b) are the calculation based on Eqs. (2)(4). The abscissa represents the configuration coordinate and the scale is adjusted to match the experimental data in column (a). The area covered by gray shading is not observed in the experiment.

stant having a dimension of length, and $H_{n}(x)$ are the Hermite functions. For calculating the WP, we have to estimate the vibrational quanta to be included. From Fig. 3, the amplitude of the first downward swing is estimated to be $0.2 \mathrm{eV}$ from the center $(0.8 \mathrm{eV})$. As the CT band edge locates at $1.4 \mathrm{eV}$ [9], the Stokes shift is estimated to be $0.6 \mathrm{eV}$, and the lattice relaxation energy to be $0.3 \mathrm{eV}$. Then the vibrational energy corresponding to the amplitude of $0.2 \mathrm{eV}$ is $0.3 / 3^{2}=0.033 \mathrm{meV}$. As the 
vibrational quantum obtained from the WP oscillation period $(\sim 300 \mathrm{fs})$ is $12.4 \mathrm{meV}$, this vibrational energy corresponds to 2.7 quanta. Subtracting 0.5 quantum for zero point vibration, the excited vibration quanta is about 2. Then for the calculation of the WP, we superpose five eigenfunctions ( $n=0$ to 4$)$ with a Gaussian weighting, that is, $c_{0}: c_{1}: c_{2}: c_{3}: c_{4}=1: 4: 6: 4: 1$. The shape of the WP calculated with this ratio almost matches the Fourier transform of the pump pulses. The instantaneous luminescence intensity at configuration coordinate $Q$ is given as

$$
I(t, Q)=I_{0}|\Psi(t, Q)|^{2} .
$$

Since $E$ is linearly related to $Q$ by Eq. (1), the luminescence spectrum directly reflects the shape of the squared amplitude of the WP on the configuration coordinate $Q$. Equation (4) can express the time-dependent shape of the WP, which is not always symmetric Gaussian. Here we show the calculated result for one period of oscillation in the upper five frames of Fig. 4(b). To reproduce the experimental results, we assumed a further broadening $0.2 \mathrm{eV}$ in calculating the spectra. The horizontal scale is adjusted to the plots of experimental data. The area covered by gray shading was not observed in the experiment. At a glance, we can notice a very close resemblance between the experiment and the calculation. At the turning points, the top of the WP goes higher and the width becomes narrower, while the WP becomes flat and low near the center of oscillation, which is in accordance with the experiment. This is because the velocity of the WP approaches zero near the turning points. The asymmetric WP shape at the turning points is also well reproduced. These good agreements unambiguously show that our experiment sees really the time-dependent shape of the WP in the harmonic potential curve. This is the first realtime capture of the WP shape for any atomic vibrations as far as the authors know, whereas the theoretical formulations and predictions for the time-dependent hot luminescence have been made as early as 1977 [11]. As the model does not involve the damping, we tried to reproduce the latter part of the oscillation by using different weighting parameters $c_{i}$. Looking at Figs. 2(a) and 4(a), one can notice that the intensity at $0.8 \mathrm{eV}$ stays almost constant after $380 \mathrm{fs}$. This means that the levels of $n \geq 2$ are not excited. If the WP had contained the components of symmetric wave functions other than $n=0$, the amplitude of the WP at the center would be modulated. Actually the WP shapes are well reproduced by a superposition of the wave functions for $n=0$ and $n=1$ with the same weight as shown in the lower four frames in Fig. 4(b).
To see the damping behavior of the "center of gravity" of the WP, we calculated the first moments of the spectra between 0.6 and $1.2 \mathrm{eV}$, and plotted them in Fig. 2(b). A large portion of the luminescence spectrum is outside the observed energy range in the first downward swing between 100 and 200 fs. However, this calculation will be good enough after $250 \mathrm{fs}$, because the area outside this energy range is estimated to be less than $15 \%$. The amplitude for each half cycle is shown in Fig. 2(a) in a logarithmic scale. The data points for the even number come lower, because the low energy part of the WP is cut from the integration. The damping in the earlier stage is estimated to be 365 fs (solid line). It is clearly seen that the decay is highly nonexponential, showing the existence of fast and slow dephasing mechanisms. It is important to note that the apparent decay time seen in the raw data in Fig. 2(a) strongly depends on the energy of observation. It is very fast at $1.2 \mathrm{eV}$ while it is rather slow at $0.9 \mathrm{eV}$. This fact shows that the observation of the total shape of the WP is inevitable even for deducing a correct decay time constant.

In summary, we have measured the time evolutions of the luminescence spectrum in quasi-1D halogen-bridged platinum complex $\left[\mathrm{Pt}(\mathrm{en})_{2}\right]\left[\mathrm{Pt}(\mathrm{en})_{2} \mathrm{Br}_{2}\right]\left(\mathrm{ClO}_{4}\right)_{4}$, and succeeded in the real-time capturing of the WP shape on the adiabatic potential surface of STE for the first time. We have found that the damping of the WP oscillation is highly nonexponential, suggesting an existence of multiple dephasing mechanisms.

[1] T. S. Rose, M. J. Rosker, and A. H. Zewail, J. Chem. Phys. 88, 6672 (1988).

[2] A. H. Zewail, J. Phys. Chem. A 104, 5660 (2000).

[3] M. Nisoli, S. D. Silvestri, O. Svelto, R. Scholz, R. Fanciulli, V. Pellegrini, F. Beltram, and F. Bassani, Phys. Rev. Lett. 77, 3463 (1996).

[4] T. Tokizaki, T. Makimura, H. Akiyama, A. Nakamura, K. Tanimura, and N. Itoh, Phys. Rev. Lett. 67, 2701 (1991).

[5] K. Toriumi, M. Yamashita, S. Kurita, I. Murase, and T. Ito, Acta Crystallogr. Sect. B 49, 497 (1993).

[6] S. Tomimoto, S. Saito, T. Suemoto, K. Sakata, J. Takeda, and S. Kurita, Phys. Rev. B 60, 7961 (1999).

[7] S. Tomimoto, S. Saito, T. Suemoto, J. Takeda, and S. Kurita, Phys. Rev. B 66, 155112 (2002).

[8] T. Matsuoka, S. Saito, J. Takeda, S. Kurita, and T. Suemoto, Nonlinear Optics 29, 587 (2002).

[9] H. Okamoto (private communication).

[10] Y. Kayanuma, J. Phys. Soc. Jpn. 57, 292 (1988).

[11] Y. Toyozawa, A. Kotani, and A. Sumi, J. Phys. Soc. Jpn. 42, 1495 (1977). 